\title{
ATRIBUTOS BIOLÓGICOS INDICADORES DA QUALIDADE DO SOLO EM SISTEMAS DE MANEJ O NA REGIÃO DO CERRADO NO SUL DO ESTADO DE GOIÁS(1)
}

\author{
A. F. D'ANDRÉ ${ }^{(2)}$, M. L. N. SILVA(3), N. CURI (4), \\ J . O. SIQUEIRA ${ }^{(4)} \&$ M. A. C. CARNEIRO ${ }^{(5)}$
}

\begin{abstract}
RESUMO
O estudo da quantidade e da atividade da biomassa microbiana pode fornecer subsídios importantes para o planejamento do uso correto da terra, considerando a natureza dinâmica dos microrganismos do solo. Este trabalho objetivou verificar alterações em atri butos biológicos indicadores da qualidade do solo na adoção de sistemas de manejo em áreas originalmente sob cerrado nativo, e selecionar os atributos com melhor desempenho em indicar tais alterações. Amostras de solo foram coletadas em três profundidades $(0-10,10-20$ e $20-40 \mathrm{~cm})$ em Latossolo Vermelho distrófico típico textura argilosa no município de Morrinhos (GO). Foram selecionadas cinco propriedades agrícolas, baseadas na sua representatividade para a região com relação ao histórico de uso e às características dos sistemas de manejo adotados. Estes consistiram de: cerrado nativo, pastagem, plantio direto, plantio direto com histórico de gradagem superficial, plantio convencional de longa duração e plantio convencional recente após pastagem. $O$ cerrado nativo foi tomado como referência, uma vez que todos os sistemas foram instalados em área originalmente de cerrado. Foram avaliados: carbono da biomassa mi crobiana ( $\mathrm{Cmic}$ ), respiração basal, quociente metabólico $\left(\mathrm{qCO}_{2}\right)$ e relação $\mathrm{Cmi} / \mathrm{CO}$. E $\mathrm{m}$ adição, foram avaliados o carbono orgânico total (CO) e alguns atributos de ferti lidade do solo. A adoção dos sistemas agrícolas e da pastagem reduziu os teores de $\mathrm{Cmic}$ na camada superficial, em relação ao cerrado nativo. Excetuando o sítio sob cerrado, o maior valor de Cmic foi observado na pastagem e o menor no plantio convencional de longa duração.
\end{abstract}

(1) Parte da Tese de Mestrado do primeiro autor, apresentada ao Departamento de Ciência do Solo, Universidade Federal de Lavras - UFLA. Trabalho apresentado no XXVIII Congresso Brasileiro de Ciência do Solo, Londrina (PR), 1 a 6 de julho de 2001. Recebido para publicação em junho de 2001 e aprovado em agosto de 2002.

(2) Professor do Centro Federal de Educação Tecnológica - CEFET de Urutai. Fazenda Palmital, Km 2,5, CEP 75790-000 Urutai (GO). E-mail: dandrea@ufla.br

(3) Professor Adjunto do Departamento de Ciência do Solo, Universidade Federal de Lavras - UFLA. Caixa Postal 37, CEP 37200000 Lavras (MG). Bolsista do CNPq. E-mail: marx@ufla.br

(4) Professor Titular do Departamento de Ciência do Solo, UFLA. Bolsista do CNPq. E-mails: niltcuri@ufla.br; siqueira@ufla.br

(5) Professor Adjunto do Centro de Ciências Agrárias e Biológicas, Campus Avançado de J atai, Universidade Federal de Goiás UFG. Br 364 Km 192, CEP 75800-000 J atai (GO). E-mail: mcarbone@jatai.ufg.ub 


\begin{abstract}
Não foram observadas diferenças si gnificati vas entre os sistemas de manejo para respiração basal e $\mathrm{qCO}_{2}$. $0 \mathrm{Cmic}$ indicou alterações significativas na instalação de sistemas de manejo em relação ao cerrado nativo e, embora tenha apontado diferenças apenas entre dois dos cinco sistemas cultivados, foi indicativo de maior equilíbrio da microbiota do solo no cerrado.
\end{abstract}

\title{
Termos de indexação: sustentabilidade, plantio convencional, plantio direto, matéria orgânica.
}

\author{
SUMMARY: BIOLOGICAL INDICATOR ATTRIBUTES OF SOIL QUALITY \\ UNDER MANAGEMENT SYSTEMS IN THE CERRADO REGION \\ OF THE SOUTHERN GOIÁS STATE, BRAZIL
}

\begin{abstract}
Due to the dynamic nature of soil microorganisms, the study of microbial biomass quantity and activity can furnish important information for the planning of adequateland use. Theobjective of this work was to verify alterations in biological attributes of soil quality indicators after the adoption of management systems in originally native cerrado areas, and to select the attributes with the best performance of indicating such alterations. Soil samples were coll lected at threedepths $(0-10,10-20$, and 20-40 cm) in a clayey texture, typic Dystrophic Red Latosol (Oxisol) in M orrinhos county, Goiás State. Fivefarms werechosen, based on their representativeness for the region in relation to their history of use and the characteristics of theadopted management systems. Thesesystems ind uded: nativecerrado, pasture, no-till, no-till with a history of superficial harrowing, long-term conventional tillage, and recent conventional tillage following pasture. The native cerrado was considered reference, onceall test areas had original ly been covered by this vegetation type Thefollowing attributes wereevaluated: carbon of microbial biomass ( $\mathrm{Cmic}$ ), basal respiration, metabol ic quotient $\left(\mathrm{qCO}_{2}\right)$ and $\mathrm{Cmic/} O \mathrm{OC}$ ratio. In addition, the total organic carbon $(\mathrm{OC})$ and some soil fertility attributes wereevaluated. Theintroduction of agricul tural systems and pasture reduced the Cmic amounts in thesurfacel ayer in compari son to thenativecerrado. Without considering thesiteunder cerrado, thehighest $\mathrm{Cmic}$ valuewas observed in the pastureand thel owest onein thelong-term conventional tillagesoil. No significant differences in basal respiration and $\mathrm{qCO}_{2}$ values were observed among the management systems. The Cmic indicated significant alterations caused by the implantation of management systems in relation to the native cerrado. Though it had revealed differences only between two of the five cul tivated systems, the $\mathrm{Cmic}$ was an indicator for a higher microbiota equilibrium in the soil under cerrado.
\end{abstract}

Index terms: sustainability, conventional tillage, no-till, soil organic matter.

\section{INTRODUÇÃO}

Os conceitos de qualidade do sol o mais difundidos são aquel es que ressaltam o seu aspecto funcional, como proposto por Doran \& Parkin (1994), que consideraram a qualidade do sol o a capacidade deste de funcionar dentro dos limites do ecossistema para sustentar a produtividade biológica, manter a qualidade ambiental e promover a saúde de plantas e animais. Esta abordagem leva em consideração não apenas o papel do sol o na produção agrícola, mas também a sua participação em funções específicas no ecossistema, das quais depende a sustentabilidade em longo prazo. A restauração da qualidade do solo após a ocorrência de processos de degradação relaciona-se diretamente com o restabelecimento dessas funções (Kimpe \& Warkentin, 1998).
No Brasil Central, grande parte da vegetação original do cerrado foi substituída por pastagens e culturas anuais (Carvalho Filho et al., 1998). Na passagem de sistemas naturais para agrícolas, muitos atributos do solo são alterados, alguns dos quais, por estarem relacionados com processos do ecossistema e serem sensíveis a variações no uso e manejo do solo, indicam al terações na sua qualidade (Doran \& Parkin, 1996). Demaneira geral, é possível obter informações bastante detalhadas sobre propriedades químicas e físicas do solo, enquanto o aspecto biol ógico é pouco conhecido. Nesse contexto, as populações de organismos do solo revelam natureza dinâmica e são facilmente afetadas por distúrbios físicos, causados pel o cultivo, ou químicos, resultantes da aplicação de fertilizantes e pesticidas (Kimpe \& Warkentin, 1998). 
A biomassa microbiana, que representa a parte viva da matéria orgânica do solo, contém, em média, de 2 a $5 \%$ do carbono orgânico, de 1 a $5 \%$ do nitrogênio orgânico e de 2 a 20 \% do fósforo orgânico nos solos tropicais (Smith \& Paul, 1990). Ela é composta por bactérias, fungos e representantes da micr ofauna, que participam de importantes funções do solo, como a ciclagem de nutrientes e energia, regulando as transformações da matéria orgânica (Turco et al., 1994).

Além de sua função catal isadora das transformações bioquímicas do solo, a biomassa microbiana representa um compartimento lábil de muitos nutrientes, que são reciclados rapidamente, com tempo de residência em torno de três meses (Duxbury et al., 1989). O carbono contido na biomassa microbiana é o destino inicial do carbono em transformação no solo e funciona como energia armazenada para processos microbianos e, por apresentar respostas rápidas a alterações no solo, pode ser utilizado como identificador precoce de alterações na matéria orgânica (Rice et al., 1996) e, assim, indicar a qualidade do solo (Dick et al., 1996).

A relação entre o carbono da bi omassa microbiana e o carbono orgânico do solo reflete processos importantes relacionados com adições e transformações da matéria orgânica, assim como a eficiência de conversão do carbono desta em carbono microbiano(Sparling, 1992). A atividadeheterotrófica da biomassa pode ser avaliada pela liberação de C$\mathrm{CO}_{2}$ em amostras coletadas no campo, sendo a quantidade de carbono liberado indicativo do carbono lábil ou prontamente metabolizável do solo (Doran \& Parkin, 1996).

A interpretação dos resultados da atividade biológica deve ser feita com critério, uma vez que el evados val ores de respi ração nem sempreindicam condições desejáveis: uma alta taxa de respiração pode significar, em curto prazo, liberação de nutrientes para as plantas e, em longo prazo, perda de carbono orgânico do sol o para a atmosfera (Parkin et al., 1996). Anderson \& Domsch (1993) propuseram a determinação do quociente metabólico, $\mathrm{qCO}_{2}$ (relação entre a quantidade de $\mathrm{CO}_{2}$ produzido por unidade de $C$ da biomassa mi crobiana e por unidade de tempo), como componente rel evante na aval iação dos efeitos ambientais e antropogênicos sobre a atividade microbiana no solo. De fato, trabalhando em sol os sob floresta, Gama-Rodrigues et al . (1997) verificaramque o $\mathrm{CCO}_{2}$ foi umindicador sensível para estimar opotencial dedecomposiçãoda matéria orgânica.

Atualmente, considerando a importância dos atributos biológicos para os processos que ocorrem no solo, verifica-se que estudos a respeito da quantidade e atividade da biomassa microbiana podem fornecer subsídios para o planejamento do uso correto da terra.

O presente trabalho teve por objetivo verificar alterações em atributos biológicos em decorrência da adoção de sistemas de manejo distintos em áreas original mente sob cerrado nativo, a fim de sel ecionar os atributos sensíveis capazes de indicar tais modificações no ecossistema, em L atossol oVermel ho distrófico típico da região sul do estado de Goiás.

\section{MATERIAL E MÉTODOS}

O trabalho foi realizado a partir de amostras de sol o col etadas no município de Morrinhos, no sul do estado de Goiás, situado na unidade geomorfológica do Planalto Central Goiano, subunidade Planalto Rebaixado de Goiânia. O solo foi desenvolvido a partir de cobertura detrito-laterítica Pleistocênica sobre micaxistos do grupo Araxá do Proterozóico Inferior (Projeto Radambrasil, 1983). O clima da região recebe a dassificação Aw (tropical estacional de savana) no sistema de Köppen, com inverno seco e verão quente, e temperatura do mês mais frio superior a $18^{\circ} \mathrm{C}$. A precipitação média da região é de $1.380 \mathrm{~mm}$, com período chuvoso de outubro a abril e período seco de maio a setembro, estando $80 \%$ das chuvas concentradas entre dezembro e março (Freitas \& Blancaneaux, 1998).

Foram selecionadas cinco propriedades agrícolas para as amostragens (uma para cada sistema de manejo), escol hidas com base na sua representatividade para a região em estudo, no que se refere ao histórico de uso da terra e às características dos sistemas de manejo adotados. Estudos anteriores realizados na microbacia piloto do estado de Goiás em Morrinhos revelaram que a maior parte dos estabelecimentos agrícolas encontra-se sob pastagens ou culturas anuais, principalmente com milho, soja earroz, o que reflete a condição existente, em geral, para a região dos cerrados (Carvalho Filho et al., 1998; Teixeira et al., 1998).

Assim, no presente estudo, foram escol hidas duas situações de plantio direto, duas de plantio convencional e uma pastagem, no município de Morrinhos. Em adição, foi sel ecionada uma área sob vegetação de cerrado nativo, empregada como referência por setratar de um sistema em equilíbrio e sem histórico de intervenção humana (Quadro 1). $\mathrm{Em}$ todos os locais em que foram coletadas as amostras, o solo pertence à unidade Latossolo Vermelho distrófico típico (EMBRAPA, 1999).

As amostragens foram efetuadas em fevereiro de 2000, em três profundidades (0-10, 10-20 e20-40 cm). Para cada profundidade, em determinado sistema de manejo, foram retiradas três amostras compostas, cada uma constituindo uma repetição, não caracterizando repetição espacial, no sentido estrito de áreas distintas e casualizadas. Por ocasião da amostragem nas áreas agrícolas cultivadas, o sistema convencional delonga duração (PC1) estava 


\section{Quadro 1. Características dos sistemas de manejo estudados em Latossolo Vermelho distrófico típico do sul de Goiás}

\begin{tabular}{|c|c|c|}
\hline Sistema & Símbolo & Histórico de uso \\
\hline Cerrado nativo & CER & $\begin{array}{l}\text { Vegetação típica de cerrado 'stricto sensu', sem histórico de interferência } \\
\text { humana em uso agrícola. }\end{array}$ \\
\hline Pastagem & PAS & $\begin{array}{l}\text { Pastagem plantada de Brachiaria decumbens de longo uso, sob pastejo } \\
\text { contínuo de gado bovino em regime extensivo e sem manejo de fertilidade } \\
\text { nos últimos } 10 \text { anos. }\end{array}$ \\
\hline Plantio direto irrigado & PD1 & $\begin{array}{l}\text { Plantio direto sob pivô central, desde o ano agrícola 1995/96, com cultivo } \\
\text { principal de milho para semente sucedido por feijão na época seca. Em } \\
\text { 1998, foi subsolado a } 15 \mathrm{~cm} \text { de profundidade para incorporação de } \\
\text { termofosfato. }\end{array}$ \\
\hline Plantio direto irrigado & PD2 & $\begin{array}{l}\text { Plantio direto sob pivô central, desde o ano agrícola 1995/96, com cultivo } \\
\text { de milho, feijão e arroz e uma safra de tomate industrial em 1998, na } \\
\text { qual foi efetuada uma gradagem superficial a } 10 \mathrm{~cm} \text { de profundidade. }\end{array}$ \\
\hline Plantio convencional irrigado & PC1 & $\begin{array}{l}\text { Plantio convencional de longa duração com uso de grade pesada, sob pivô } \\
\text { central desde o ano agrícola 1998/99, com cultivo de milho após mais de } \\
15 \text { anos de rotação soja/milho na condição de sequeiro. }\end{array}$ \\
\hline Plantio convencional irrigado & PC2 & $\begin{array}{l}\text { Plantio convencional recente sob pivô central e uso de grade pesada, } \\
\text { desde o ano agrícola 1998/99, com histórico de rotação de culturas com } \\
\text { abóbora/feijão/milho-doce, após mais de } 10 \text { anos sob pastagem de } \\
\text { Brachiaria decumbens. }\end{array}$ \\
\hline
\end{tabular}

na fase final do ciclo da cultura, enquanto os demais encontravam-se em fase posterior à colheita. As amostras de solo foram acondicionadas em sacos plásticos, protegidas da luz e mantidas em caixas térmicas, sendo transportadas para olaboratório no prazo máximo de 48 horas após a coleta. As amostras foram peneiradas ( $2 \mathrm{~mm}$ ) ea umidade ajustada para 50 a $60 \%$ da capacidade de campo, sendo acondicionadas em sacos plásticos com suspiros e mantidas sob refrigeração $\left(4{ }^{\circ} \mathrm{C}\right)$ atéà realização das análises de biomassa e respiração. Antes desse procedimento, foram retiradas subamostras para as análises químicas e físicas.

A análise granulométrica foi efetuada pelo método da pipeta (Day, 1965), após dispersão da amostra com NaOH 1 mol L-1 e agitação rápida (6.000 rpm) por 15 minutos. Em todos os sistemas de manejo estudados, a composição granul ométrica remeteu o solo à classe textural argilosa (Quadro 2). $\mathrm{O} \mathrm{pH}$ em água, os componentes do complexo sortivo e o fósforo disponível foram determinados segundo Vettori (1969) e EMBRAPA (1997), enquanto o carbono orgânicototal foi determinado por oxidação a quente com dicromato de potássio e titulação com sulfato ferroso amoniacal (Walkley \& Black, 1934).

O carbono da biomassa microbiana foi avaliado pelo método da fumigação-extração (Vance et al., 1987), sendo determinado após o ataque das amostras com clorofórmio, que provoca morte dos microrganismos e liberação dos componentes celulares. O carbono de $25 \mathrm{~g}$ de solo de amostras fumigadas e não fumigadas foi extraído com $\mathrm{K}_{2} \mathrm{SO}_{4}$ $0,5 \mathrm{~mol} \mathrm{~L}^{-1}$ e a uma alíquota desse extrato foram adicionados $\mathrm{K}_{2} \mathrm{Cr}_{2} \mathrm{O}_{7} 0,0667 \mathrm{~mol} \mathrm{~L}^{-1} \mathrm{e} \mathrm{H}_{2} \mathrm{SO}_{4} / \mathrm{H}_{3} \mathrm{PO}_{4}$ (1:2), com aquecimento. A titulação foi feita com sol ução de sulfato ferroso amoniacal $0,0333 \mathrm{~mol} \mathrm{~L}^{-1}$. O carbono da biomassa microbiana foi calculado a partir da seguinte expressão:

$$
\mathrm{Cmic}=(\mathrm{F}-\mathrm{NF}) / \mathrm{Kec} \text {, }
$$

sendo Cmic: carbono da biomassa microbiana $\left(\mu \mathrm{g} \mathrm{g}^{-1}\right)$;

$\mathrm{F}: \mu \mathrm{g} \mathrm{g}^{-1}$ de carbono da amostra fumigada;

$\mathrm{NF}: \mu_{\mathrm{g} \mathrm{g}}{ }^{-1}$ de carbono da amostra não fumigada;

Kec: fator para converter o carbono extraído a Cmic (foi usado 0,30, ovalor mais freqüenteencontrado por Feigl et al., 1995).

O quociente microbiano, ou relação $\mathrm{Cmic/CO}$, foi calculado de acordo com Sparling (1992), pela expressão:

$$
\mathrm{Cmic} / \mathrm{CO}=(\mathrm{Cmic}) /(\mathrm{CO}) / 10
$$

sendo Cmic/CO: quociente microbiano ( \%);

Cmic: carbono da biomassa microbiana $\left(\mu \mathrm{g} \mathrm{g}^{-1}\right)$;

CO: carbono orgânico total $\left(\mathrm{g} \mathrm{kg}^{-1}\right)$. 
Quadro 2. Caracterização de um Latossolo Vermelho distrófico típico, para seis diferentes situações de manejo do solo

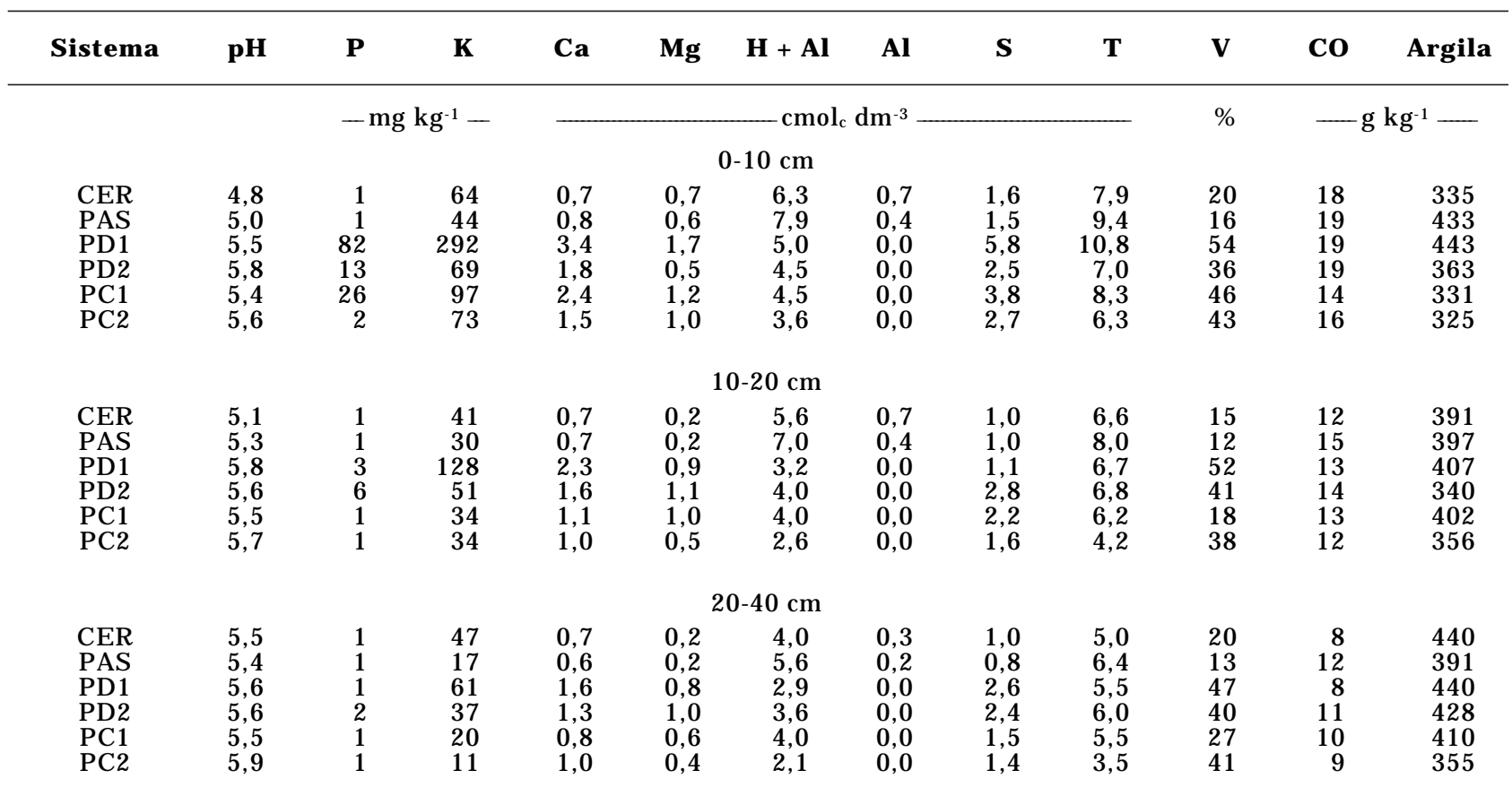

CER: cerrado nativo; PAS: pastagem; PD1: plantio direto; PD2: plantio direto com histórico de gradagem superficial; PC1: plantio convencional de longa duração; PC2: plantio convencional recente após pastagem. S: soma de bases; T: capacidade de troca catiônica; V: saturação por bases; CO: carbono orgânico total.

A taxa de respiração basal foi estimada pelo $\mathrm{CO}_{2}$ liberado a partir de $20 \mathrm{~g}$ de solo durante incubação por $72 \mathrm{~h} . \mathrm{O} \mathrm{CO}_{2}$ foi capturado por solução de $\mathrm{NaOH}$ $0,05 \mathrm{~mol} \mathrm{~L}^{-1}$ e determinado por titulação com HCl $0,05 \mathrm{~mol} \mathrm{~L}^{-1}$ (Isermeyer, 1952 citado por Alef \& Nanni pieri, 1995). O quociente metabólico $\left(\mathrm{qCO}_{2}\right)$ foi cal culado pela razão entrea taxa de respiração basal e o carbono da biomassa microbiana (Anderson \& Domsch, 1993), sendo expresso em $\mu \mathrm{g} \mathrm{CO} 2 \mu \mathrm{g}$ Cmic ${ }^{1}$ dia $^{-1}$.

O delineamento experimental utilizado foi o inteiramente casualizado, com estrutura em faixas de acordo com as profundidades de amostragem. Para verificar os pressupostos da análise de variância (aditividade do model o e normalidade de distribuição dos erros), foram utilizados os procedimentos "Univariate" e "General Linear Models" do pacote estatístico SAS (SAS, 1985). Os resultados foram submeti dos à análise de variância para verificação dos efeitos dos sistemas de manejo, da profundidade e da interação sistema $x$ profundidade. Os val ores de carbono orgânico total, respiração basal e quociente metabólico $\left(\mathrm{qCO}_{2}\right)$ foram transformados com o uso da função $y=\log x$ (logaritmo base 10) para as análises de variância. As comparações múltiplas de médias foram feitas pelo teste de Tukey a $5 \%$, utilizando o programa SISVAR (Ferreira, 2000). Após esse procedimento, os valores desses atributos foram novamente transformados com o uso da função y $=10^{x}$ para apresentação dos resultados.

\section{RESULTADOS E DISCUSSÃO}

\section{Carbono da biomassa microbiana}

O carbono da biomassa mi crobiana (Cmic) variou nas diferentes condições estudadas e também em profundidade (Figura 1). Diferenças significativas entre os sistemas de manejo foram observadas apenas na camada superficial, onde são mais intensos os processos de transformação da matéria orgânica pelos microrganismos do solo. Nessa profundidade, o cerrado nativo (CER) apresentou um teor el evado de Cmic (cerca de $1.310 \mathrm{\mu g} \mathrm{g}^{-1}$ ), significativamente mai or do que os valores obtidos para os demais sistemas. Dentre estes, o carbono da biomassa mi crobiana na pastagem de braquiária (PAS) foi de $666,2 \mu^{g^{-1}}$ e superou o valor relativo ao sistema convencional PC1, que foi de $213,4 \mu \mathrm{g} \mathrm{g}^{-1}$.

Tomando o sistema sob vegetação de cerrado nativo como referência, observou-se uma redução nos valores de Cmic da ordem de $49 \%$, para o sistema pastagem, e de $73 \%$, para a média dos sistemas com 
C BIOMASSA MICROBIANA RELAÇÃO Cmic/CO

$0-10 \mathrm{~cm}$
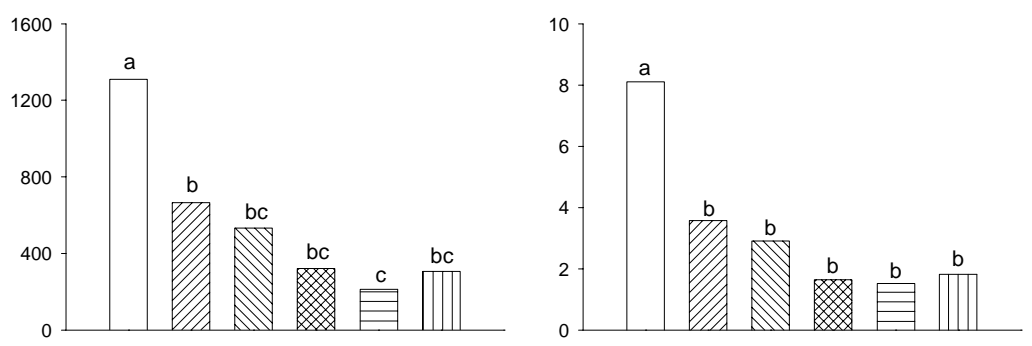

$10-20 \mathrm{~cm}$
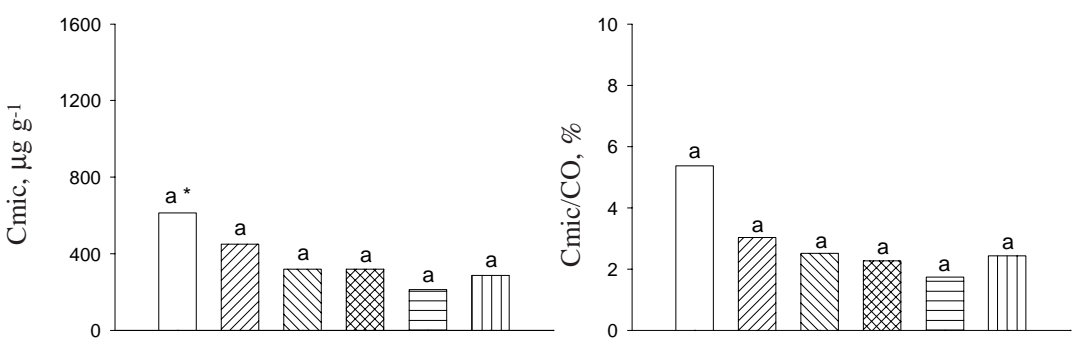

$20-40 \mathrm{~cm}$
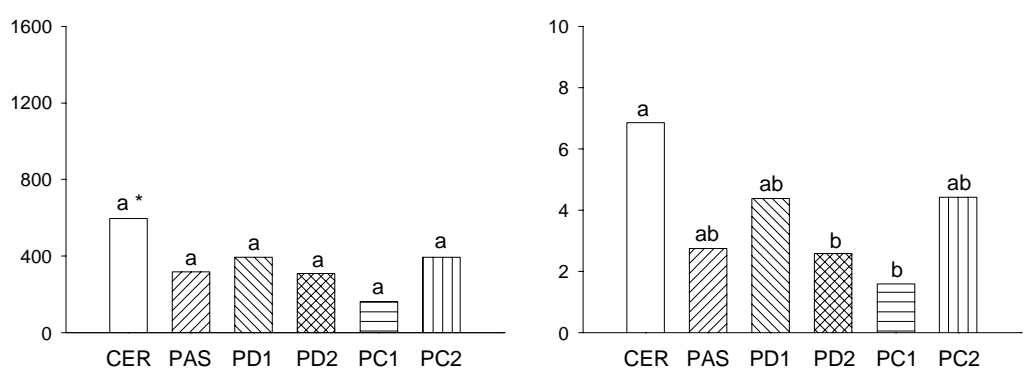

Figura 1. Carbono da biomassa microbiana $(\mathrm{Cmic})$ e relação $\mathrm{Cmic} /$ carbono orgânico total $(\mathrm{Cmic} / \mathrm{CO})$, para seis sistemas de manejo e três profundidades. Para ambos os atributos, dentro de cada profundidade, médias seguidas da mesma letra não diferem pelo teste de Tukey, a $5 \%$. CER : cerrado nativo; PAS: pastagem; PD1: plantio direto; PD2: plantio direto com histórico de gradagem superficial; PC1: plantio convencional de longa duração; PC2: plantio convencional recente após pastagem. * significativamente menor que na profundidade de $0-10 \mathrm{~cm}$.

culturas anuais (sítios sob plantio direto e convencional). A pesar denãoterem sido observadas diferenças significativas, o teor de Cmic na profundidade de $10-20 \mathrm{~cm}$ seguiu a mesma tendência da camada superficial. O mesmo padrão, no entanto, não foi observado na camada mais profunda do solo (20-40 cm) (Figura 1).

Resultados semel hantes aos deste trabal ho foram reportados por Mendes et al. (1999), em Latossolo Vermelho-Amarelo dos cerrados, com teores mais elevados de Cmic no solo sob vegetação nativa do que em sistemas cultivados, o mesmo ocorrendo nos estudos de Venzke Filho (1999), em Latossolo Vermel ho-Escuro no Paraná. Valores de Cmic mais baixos no sistema pastagem (PAS) em relação ao cerrado nativo (CER) seguem a tendência dos resultados obtidos por Fernandes (1999) em solos da Amazônia. O autor verificou que, após a retirada da vegetação nativa e estabel ecimento da pastagem, houve aumento nos teores de $\mathrm{Cmic}$ durante os cinco primeiros anos, mantendo-se num patamar de estabilidadeaté 20 anos após a alteração da condi ção inicial. A partir deste período, esse autor observou reduções no $\mathrm{Cmic}$, decorrentes das condições de degradação da pastagem adotada. Estudos de Marchiori J únior \& M elo (1999) mostraram maiores teores de Cmic em solo sob pastagens do que sob culturas anuais, do mesmo modo que o observado no presente trabal ho entre os sistemas PAS e PC1.

O val or de biomassa microbiana mais el evado no cerrado nativo é reflexo de uma situação bastante particular para a microbiota do solo nesse sistema, 
que é estimulada pelo fornecimento contínuo de materiais orgânicos com diferentes graus de susceptibilidade à decomposição, originados da vegetação. A diversidade das espécies vegetais do cerrado nativo, notadamente maior do que a dos demais sistemas de manejo avaliados, implica a deposição de substratos orgânicos oxidáveis com composição variada na serapilheira. Além disso, existe maior diversidade de compostos orgânicos depositados na rizosfera, o que constitui fator favorável à sobrevivência e crescimento dos diferentes grupos de microrganismos do solo. Neste sentido, a abundância de microrganismos decompositores pode contribuir para estimular também a microfauna saprófita e predadora destes, que é constituída por protozoários, nematóides e microartrópodos. Assim, as condições distintas do sol o sob vegetação de cerrado nativo, juntamentecom a ausência de perturbações decorrentes de atividade antrópica, tornam possível a existência de maiores teores de biomassa microbiana, indicando o maior equilíbrio da microbiota do sol o nesse ecossistema.

No sistema pastagem (PAS), o carbono da biomassa microbiana não foi tão alto como no cerrado nativo, mas superou a média dos sistemas com culturas anuais efoi significativamente maior do que o sistema PC1 (Figura 1). Apesar da baixa diversidade de cobertura vegetal existente no sistema PAS (seu histórico de uso indica uma condição de pastagem plantada de Brachiaria decumbens de longa duração- Quadro 1), a biomassa microbiana foi, provavelmente, estimulada em decorrência do elevado efeito rizosférico da gramínea, amplamente documentado na literatura (Rovira, 1978; Alexander, 1980; Reid \& Goss, 1980), com a liberação de substâncias orgânicas diversas (exsudatos, mucilagens, secreções) por um sistema radicular denso e de constanterenovação, com maior disponibilidade de nutrientes para a microbiota do solo no ambiente rizosférico.

Com relação ao plantio convencional $\mathrm{PC} 1$, os resultados indicam que as características desse sistema de manejo (alto revolvimento eapenas duas espécies de culturas anuais por mais de 15 anos de cultivo) contribuem para reduzir a quantidade e, possivelmente, a diversidade dos microrganismos, com grande impacto na microbiota do solo. Acreditase que as adi ções de carbono oxidável nesse sistema não estejam sendo suficientes para atender à demanda para a manutenção da biomassa existente, o que leva ao decréscimo acentuado observado nos valores de Cmic.

No presente estudo, não houve diferenças significativas nos teores de carbono da biomassa microbiana presentes no sol o sob pastagem (PAS) e sob três sistemas com culturas anuais (PD1, PD2 e PC2), ao contrário do reportado para o PC1. É possível que se encontre uma explicação para esse fato ao considerar as difer enças no tempo de adoção dos diversos sistemas estudados. Verifica-se que, dos quatro sistemas com culturas anuais, apenas o PC1 apresenta longo tempo de implantação (Quadro 1), sugerindo que ainda estejam ocorrendo alterações na biomassa microbiana dos sistemas recentes, as quais devem estender-se até que um novo nível de equilíbrio seja atingido.

Nesse sentido, comparando sistemas agrícolas, Balota et al. (1998) verificaram um aumento nos teores de Cmic no plantio direto em relação a sistemas convencionais, em experimento com 16 anos de duração, do mesmo modo que Carvalho (1997), em experimento deseteanos no Sul do Brasil. Todavia, Mendes et al. (1999) não observaram diferenças significativas no $\mathrm{Cmic}$, em sol os da região dos Cerrados, entre um sistema de plantio direto com dois anos de implantação e um convencional.

Estes resultados estão de acordo com os obtidos no presente estudo, uma vez que são recentes (quatro a cinco anos) os sistemas de plantio direto avaliados neste trabalho. Para Vasconcellos et al. (1999), a biomassa microbiana imobiliza mais carbono quando resíduos vegetais são deixados na superfície. Com isso, é possível esperar que, com o decorrer do tempo de cultivo, os sítios sob plantio direto apresentem aumento nos valores de $\mathrm{Cmic}$, principalmente na camada de $0-10 \mathrm{~cm}$, ondea média deste atributo nos dois sistemas deplantio direto $\left(428,5 \mu^{-1} \mathrm{~g} \mathrm{~g}^{-1}\right)$ superou a média dos convencionais $\left(260,6 \mu \mathrm{g} \mathrm{g}^{-1}\right)$, ainda que a diferença entre os sistemas não tenha sido significativa.

O efeito da profundidade sobre o $\mathrm{Cmic}$ foi significativoapenas no cerrado nativo (CER), no qual maiores valores de $\mathrm{Cmic}$ foram encontrados na camada de $0-10 \mathrm{~cm}$ em relação às subjacentes (Figura 1 e análise de variância). Nos demais sistemas, o Cmic apresentou valores relativamente constantes ao longo do perfil, não havendo variação significativa desse atributo em profundidade.

\section{Relação Cmic/CO}

A distribuição do quociente microbiano (relação Cmic/CO) seguiu a mesma tendência apresentada pel os resultados de carbono da biomassa microbiana, principalmente na camada superficial do solo (Figura 1). A contribuição do carbono da biomassa microbiana para o carbono orgânico total do solo variou, na camada de $0-10 \mathrm{~cm}$, de 1,52 a $8,10 \%$. O maior valor, referente ao cerrado, destacou-se daqueles cal culados para todos os demais sistemas (Figura 1).

Em trabalho desenvolvido na Nova Zelândia, Sparling (1992) observou uma relação Cmic/CO maior em pastagens, quando comparadas com florestas nativas ou exóticas. No Brasil, em experimento realizado por Geral des et al. (1995), na Amazônia, a relação $\mathrm{Cmic/CO}$ foi mais el evada em pastagem de quatroanos do quena mata natural. No 
entanto, com oaumento do tempo de estabel eci mento da pastagem, os autores observaram um decréscimo na relação, indicando redução da participação do carbono da biomassa microbiana em relação ao carbono orgânico total. Nesse experimento, o solo sob pastagem por 15 anos apresentou valores de $\mathrm{Cmi} / \mathrm{CO}$ menores do que aqueles sob floresta, o que foi atribuído a modificações causadas pelo pisoteio dos animais.

No presente trabalho, a tendência apresentada por Geraldes et al. (1995) para as florestas amazônicas também foi observada, uma vez que os val ores de $\mathrm{Cmi} / \mathrm{CO}$ cal culados no sistema pastagem foram significativamente menores do que no cerrado nativo (Figura 1 ).

Com relação aos sistemas agrícolas, não se observou uma distribuição diferenciada da relação $\mathrm{Cmic/CO}$ entre os dois sistemas de plantio (direto e convencional), o que vai de encontro aos resultados reportados por Bal ota et al . (1998), que verificaram valores de $\mathrm{Cmic/CO}$ mais el evados no plantio direto do que em sistema convencional.

Entretanto, segundo Sparling (1992), a razão $\mathrm{Cmic/CO}$ éinfluenciada por diversos fatores, como o grau de estabilização do carbono orgânico e o histórico de manejo do solo, alertando que, para se saber o quanto a razão Cmic/CO está em equilíbrio ou se encontra ainda em fase de degradação ou recuperação, énecessário estabelecer um val or base para cada situação particular, sendo aparentemente pouco extrapoláveis para condições distintas tais valores de referência. O autor observou que a recuperação dos teores de carbono da biomassa microbiana após o reestabel ecimento de pastagens em área de cultivos anuais foi rápida eque, em razão dos baixos teores de CO nos solos sob cultivo, a relação $\mathrm{Cmic/CO}$ atingiu os níveis da pastagem permanenteapós apenas dois ou três anos. Por esse motivo, a proporção de carbono da biomassa microbiana em relação ao carbono orgânico do solo não pode ser considerada um indicador da recuperação do estado da matéria orgânica nos solos cultivados, caso seja analisada isoladamente.

\section{Respiração basal e quociente metabólico $(\mathrm{qCO})$}

Não foram verificadas diferenças significativas nos valores da respiração basal entre os vários sistemas de manejo, o mesmo ocorrendo com o $\mathrm{qCO}_{2}$ (Quadro 3). No entanto, para esse atributo, houve efeito de profundidade, e os valores médios da camada de $20-40 \mathrm{~cm}$ superaram os da camada superficial do solo.

A influência do $\mathrm{pH}$ do solo sobre o quociente metabólico foi estudada por Anderson \& Domsch (1993), em experimento envolvendo diversos sítios florestais. Os autores verificaram que, na camada superficial, valores baixos de $\mathrm{pH}$ reduziram a biomassa microbiana e a produção de $\mathrm{C}-\mathrm{CO}_{2}$ em
Quadro 3. Respiração basal e quociente metabólico $\left(\mathrm{qCO}_{2}\right)$ em Latossolo Vermelho distrófico típico, para seis sistemas de manejo

\begin{tabular}{|c|c|c|c|}
\hline \multirow{2}{*}{ Sistema } & \multicolumn{3}{|c|}{ Profundidade $(\mathrm{cm})$} \\
\hline & $0-10$ & $10-20$ & $20-40$ \\
\hline & \multicolumn{3}{|c|}{ Respiração basal, $\mu \mathrm{g} \mathrm{CO}_{2} \mathrm{~g}^{-1} \mathrm{~h}^{-1}$} \\
\hline $\begin{array}{l}\text { CER } \\
\text { PAS } \\
\text { PD1 } \\
\text { PD2 } \\
\text { PC1 } \\
\text { PC2 }\end{array}$ & $\begin{array}{l}19,41 \\
17,97 \\
12,83 \\
21,20 \\
18,25 \\
17,99\end{array}$ & $\begin{array}{l}24,78 \\
10,89 \\
11,89 \\
26,55 \\
19,73 \\
35,06\end{array}$ & $\begin{array}{l}25,64 \\
20,87 \\
17,96 \\
22,35 \\
27,74 \\
44,26\end{array}$ \\
\hline Média & $\begin{array}{r}17,94 \mathrm{a} \\
\mathrm{qCO}_{2}\end{array}$ & $\begin{aligned} & 21,48 \mathrm{a} \\
& \mathrm{C}_{2} \mu \mathrm{g} \mathrm{Cmi}\end{aligned}$ & $\begin{array}{l}26,47 \text { a } \\
\text { dia-1 }\end{array}$ \\
\hline $\begin{array}{l}\text { CER } \\
\text { PAS } \\
\text { PD1 } \\
\text { PD2 } \\
\text { PC1 } \\
\text { PC2 }\end{array}$ & $\begin{array}{l}0,36 \\
0,72 \\
0,58 \\
1,76 \\
2,14 \\
1,48\end{array}$ & $\begin{array}{l}1,06 \\
0,59 \\
0,91 \\
2,13 \\
2,31 \\
2,97\end{array}$ & $\begin{array}{l}1,09 \\
1,62 \\
1,23 \\
1,88 \\
4,81 \\
3,22\end{array}$ \\
\hline Média & $1,17 \mathrm{~b}$ & $1,66 a b$ & 2,31 \\
\hline
\end{tabular}

CER: cerrado nativo; PAS: pastagem; PD1: plantio direto; PD2: plantio direto com histórico de gradagem superficial; PC1: plantio convencional de Ionga duração; PC2: plantio convencional recente após pastagem. Médias seguidas pela mesma letra não diferem entre si pelo teste de Tukey, a $5 \%$.

relação à faixa neutra de $\mathrm{pH}$. No entanto, os val ores de $\mathrm{qCO}_{2}$ foram maiores sob condição ácida, indicando aos autores uma maior liberação de $\mathrm{C}-\mathrm{CO}_{2}$ por unidade de $\mathrm{Cmic}$.

No presente estudo, apesar de terem sido verificadas diferenças no $\mathrm{pH}$ do solo na camada superficial (Quadro 2 e análise de variância), não foram detectadas alterações na respiração do solo ou no quociente metabólico que refletissem essa condição. Pelo contrário, os sistemas com maior pH tenderam a apresentar os maiores valores de $\mathrm{qCO}_{2}$, ainda que a diferença não tenha sido significativa. Deve-se considerar, no entanto, quea condiçãoácida reportada por Anderson \& Domsch (1993) refere-se a uma faixa muito menor de $\mathrm{pH}(\mathrm{pH} \mathrm{KCl}$ variando em torno de 2,7 a 3,2) e que as condições de clima temperado são bastante dissimilares às dos trópicos.

No que se refere a sistemas agrícolas, Doran (1980) relatou que, em condições de pouco revolvimento do solo, como no plantio direto, existem melhores condições para o desenvolvimento das populações microbianas do que nos sistemas convencionais, na camada superficial. No caso dos sistemas convencionais, o revolvimento sistemático do solo contribui para provocar perturbações promotoras de estresse na população microbiana e, uma vez que as adições de carbono nesses sistemas 
são menores, os microrganismos terminam por consumir o carbono orgânico do solo, causando sua redução. Nesse sentido, maiores valores de $\mathrm{qCO}_{2}$ indicam que a população microbiana está consumindo mais carbono oxidável para a sua manutenção (Anderson \& Domsch, 1993).

No plantio direto, aumentos na respiração do solo têm sido justificados pelo acúmulo de matéria orgânica rica em frações lábeis à superfície (Vargas \& Scholles, 2000). Estudos desistemas convencionais e de plantio direto, em Londrina (PR), mostraram valores mais el evados de respiração basal no plantio direto, o que foi atribuído à mai or atividade bi ológica nesse sistema (Balota et al., 1998). No mesmo experimento, foram obtidos menores valores de $\mathrm{qCO}_{2}$ no plantio direto em relação aos sistemas convencionais, do mesmo modo que em outros trabalhos desenvolvidos na região Sul (Silva-Filho \& Vidor, 1984; Lima et al., 1994).

No presente estudo, este comportamento não foi verificado, uma vez que as diferenças na respiração basal e no $\mathrm{qCO}_{2}$ entre os sistemas de plantio direto e os convencionais não foram significativas. No entanto, é importante considerar que as áreas sob plantio di reto envol vidas nestetrabalho são recentes (entre quatro a cinco anos) e que a biomassa microbiana pode estar ainda em adaptação às condições do solo. Além disso, diferenças climáticas entre os Cerrados ea região Sul do País influenciam diretamente a dinâmica de carbono no ecossistema e o comportamento e atividade dos microrganismos do solo, motivo pel o qual é possível supor que venha a ocorrer maior diferenciação entre os valores de $\mathrm{qCO}_{2}$ dos sistemas convencionais e de plantio direto, ao longo do tempo.

Por fim, deve-se levar em consideração que apenas de 15 a $30 \%$ da biomassa microbiana do solo é catabolicamente ativa (Mac Donald, 1986) e queo restante dos microrganismos do solo ocorre em formas latentes ou inativas, com baixa atividade metabólica (Moreira \& Siqueira, 2002), o que pode causar dificuldades adicionais na inter pretação dos resultados do $\mathrm{qCO}_{2}$, já que em seu cál culo é levado em conta o teor total de carbono da biomassa microbiana do solo.

Uma das aproximações propostas para estabelecer critérios de avaliação da qualidade do solo é a comparação efetuada com o solo no seu estado natural (Granatstein \& Bezdicek, 1992). No presente estudo, o cerrado nativo foi estabelecido como referência para a maior parte das comparações entre sistemas de manejo. Considerando que os atributos de qualidade do solo devem ter sensibilidade o suficiente para indicar alterações decorrentes do uso, é possível verificar que o carbono da biomassa microbiana apresentou o melhor desempenho para tal. Por esse motivo, esse atributo é bastante útil em estudos a respeito da sustentabilidade de ecossistemas e pode ser indicado como um componente potencial de um índice de qualidade do solo a ser desenvolvido para fins de monitoramento das atividades agrícolas na região. O carbono da biomassa microbiana está entre os atributos citados como de grande importância em estudos da qualidade do solo, tendo sido sel ecionado por Islam \& Weil (2000) como um dos mais promissores para inclusão em índices de qualidade do sol o, por ser influenciado de maneira diferenciada na maioria das comparações entre sistemas tidos como conservacionistas ou causadores de degradação.

\section{CONCLUSÕES}

1. A instalação de pastagens esistemas demanejo agrícola em área de cerrado nativo reduziu os teores de carbono da biomassa microbiana na camada superficial do solo. Essa redução foi menor no sistema pastagem e maior no sistema agrícola convencional, com mais de 15 anos de instalação.

2. A atividade microbiana, avaliada por meio da respiração basal e do quociente metabólico $\left(\mathrm{qCO}_{2}\right)$, não diferiu entre o cerrado nativo e os diferentes sistemas de manejo estudados.

3. A profundidade exerceu efeito sobre o carbono da biomassa microbiana (maiores val ores na camada superficial do sol o sob cerrado nativo em relação às subjacentes) esobre o quociente metabólico (valores crescentes em profundidade).

4. O carbono da biomassa microbiana indicou alterações significativas decorrentes da adoção de sistemas de manejo em relação ao cerrado nativo, embora, entre os mesmos, tenha apontado apenas diferenças entre a pastagem e o sistema convencional delonga duração.

\section{AGRADE CIMENTOS}

Ao Consel ho Nacional de Desenvolvimento Científico e Tecnológico - CNPq, pelo auxílio financeiro ao projeto. Aos produtores rurais do sul do estado de Goiás, pelas facilidades concedidas durante os trabalhos de campo e pela permissão de uso das áreas cultivadas, as quais serviram de base para o desenvolvimento deste projeto.

\section{LITERATURA CITADA}

ALEF, K. \& NANNIPIERI, P., eds. Methods in applied soil microbiology and biochemistry. London, Academic Press, 1995. 576p. 
ALEXANDER, M. Introducción a la microbiologia del suelo. México, D.F., Libros y Editoriales, 1980. 491p.

ANDERSON, J.P.E. \& DOMSCH, K.H. The metabolic quotient $\left(\mathrm{qCO}_{2}\right)$ as a specific activity parameter to assess the effects of environmental conditions, such as $\mathrm{pH}$, on the microbial biomass of forest soils. Soil Biol. Biochem., 25:393-395, 1993.

BALOTA, E.L.; COLOZZI-FILHO, A.; ANDRADE, D.S. \& HUNGRIA, M. Biomassa microbiana e sua atividade em solos sob diferentes sistemas de preparo e sucessão de culturas. R. Bras. Ci. Solo, 22:641-649, 1998.

CARVALHO FILHO, A.; MOTTA, P.E.F.; CHAGAS, C. S.; KER, J.C.; BLANCANEAUX, P.; CARVALHO J r., W.; AMABILE, R.F.; COSTA, L.D. \& PEREIRA, N.R. A cobertura pedológica eas interações com as rochas, o rel evo ea cobertura vegetal. In: BLANCANEAUX, P., ed. I nterações ambientais no Cerrado: microbacia piloto de Morrinhos, estado de Goiás, Brasil. Brasília, Empresa Brasileira de Pesquisa Agropecuária, 1998. p.69-143.

CARVALHO, Y. Densidade e atividade dos microrganismos do sol o em plantio direto e convencional na região de Carambeí (PR). Curitiba, UniversidadeFederal do Paraná, 1997. 108p. (Tese de Mestrado)

DAY, P.R. Particle fractionation and particle-size analysis. In: BLACK, C.A. Methods of soil analysis. Madison, American Society of Agronomy, 1965. v.1, p.545-566.

DICK, R.P.; BREAKWELL, D.P. \& TURCO, R.F. Soil enzyme activities and biodiversity measurements as integrative microbiological indicators. In: DORAN, J.W. \& J ONES, A.J ., eds. Methods for assessing soil quality. Madison, Soil Science Society of America, 1996. p.247-272. (SSSA Special Publication, 49)

DORAN, J.W. Soil microbial and biochemical changes associated with reduced tillage. Soil Sci. Soc. Am. J ., 44:765-771, 1980.

DORAN, J.W. \& PARKIN,T.B. Defining and assessing soil quality. In: DORAN, J.W.; COLEMAN, D.C.; BEZDICEK, D.F.; STEWART, B.A., eds. Defining soil quality for a sustainable environment. Madison, Soil Science Society of America, 1994. p.3-21. (SSSA Special Publication, 35)

DORAN, J.W. \& PARKIN, T.B. Quantitative indicators of soil quality: a minimum data set. In: DORAN, J.W \& J ONES, A.J ., eds. Methods for assessing soil quality. Madison, Soil Science Society of America, 1996. p.25-37. (SSSA Special Publication, 49)

DUXBURY, J.M.; SMITH, M.S.; DORAN, J.W; J ORDAN, C.; SZOTT, L. \& VANCE, E. Soil organic matter as a source and sink of plant nutrients. In.: COLEMAN, D.C.; OADES, J.M. \& UEHARA, G., eds. Dynamics of soil organic matter in tropical ecosystems. Honolulu, Niftal Project, 1989. p.3367.

EMPRESA BRASILEIRA DE PESQUISA AGROPECUÁRIA EMBRAPA. Centro Nacional de Pesquisa de Solos. Sistema brasileiro de classificação de sol os. Brasília, Rio de J aneiro, 1999. 412p.

EMPRESA BRASILEIRA DE PESQUISA AGROPECUÁRIA EMBRAPA. Serviço Nacional de Levantamento e Conservação de Solo. Manual de métodos de análise de solo. Rio de J aneiro, Ministério da Agricultura, 1997. 212p.
FEIGL, B.J .; SPARLING, G.P.; ROSS, D.J . \& CERRI, C.C. Soil microbial biomass in Amazonian soils: evaluation of methods and estimates of pool sizes. Soil Biol. Biochem., 27:1467-1472, 1995.

FERNANDES, S.A.P. Propriedades do solo na conversão de floresta em pastagem fertilizada e não fertilizada com fósforo na Amazônia (Rondônia). Piracicaba, U niversidade de São Paulo, 1999. 131p. (Tese de Mestrado)

FERREIRA, D.F. Análises estatísticas por meio do SISVAR (Sistema para análise de variância) para Windows versão 4.0. In: REUNIÃO ANUAL DA REGIÃO BRASILEIRA DA SOCIEDADE INTERNACIONAL DE BIOMETRIA, 45., São Carlos, 2000. Anais. São Carlos, Universidade de São Carlos, 2000. p.255-258.

FREITAS, P.L. \& BLANCANEAUX, P. Avaliação sobre os aspectos climatológi cos e hidrológi cos da microbacia piloto de Goiás, Morrinhos. In.: BLANCANEAUX, P., ed. Interações ambientais no Cerrado: microbacia piloto de Morrinhos, estado de Goiás, Brasil. Brasília, Empresa Brasileira de Pesquisa Agropecuária, 1998, p.31-52.

GAMA-RODRIGUES, E.F.; GAMA-RODRIGUES, A.C. \& BARROS, N.F. Biomassa microbiana de carbono e de nitrogênio de solos sob diferentes coberturas florestais. R. Bras. Ci. Solo, 21: 361-365, 1997.

GERALDES, A.P.A.; CERRI, C.C. \& FEIGL, B.J . Biomassa microbiana de solo sob pastagens na Amazônia. R. Bras. Ci. Solo, 19:55-60, 1995.

GRANATSTEIN, D. \& BEZDICEK, D.F. The need for a soil quality index: Iocal and regional perspectives. Am. J . Altern. Agric., 7:12-16, 1992.

ISLAM, K.R. \& WEIL, R.R. Soil quality indicator properties in mid-Atlantic soils as influenced by conservation management. J . Soil Water Conserv., 55:69-79, 2000.

KIMPE, C.R. \& WARKENTIN, B.P. Soil functions and the future of natural resourses. In.: BLUME, H.P.; EGER, H.; FLEISHHAUER, E.; HEBEL, A.; REIJ, C. \& STEINER, K.G., eds. Towards sustainable land use - Furthering cooperation between people and institutions. Advances Geoecol., 31:3-10, 1998.

LIMA, V.C.; LIMA, J.M.J .C.; EDUARDO, B.J .F.P. \& CERRI, C.C. Conteúdo de carbono e biomassa microbiana em agrossistemas: comparação entre métodos de preparo do solo. Agrárias, 13:297-302, 1994.

MaC DONALD, R.M. Extraction of microorganisms from soil. Biol. Agric. Hortic., 3:361-365, 1986.

MARCHIORI J ÚNIOR, M. \& MELO, W.J . Carbono, carbono da biomassa microbiana e atividade enzimática em um solo sob mata natural, pastagem ecultura do al godoei ro. R. Bras. Ci. Solo, 23: 257-263, 1999.

MENDES, I.C.; CARNEIRO, R.G.; CARVALHO, A.M.; VIVALDI, L. \& VARGAS, M.A.T. Biomassa C e atividade microbiana em solos de cerrado sob plantio direto e plantio convencional. Planaltina, 1999. 5p. (Pesquisa em andamento - EMBRAPA Cerrados, 5)

MOREIRA, F.M.S. \& SIQUEIRA, J .O. Microbiologia ebioquímica do solo. Lavras, Universidade Federal de Lavras, 2002. $625 \mathrm{p}$. 
PARKIN, T.B.; DORAN, J.W. \& FRANCO-VIZCAÍNO, E. Field and laboratory tests of soil respiration. In: DORAN, J.W. \& J ONES, A.J., eds. Methods for assessing soil quality. Madison, Soil Science Society of America, 1996. p.231-245. (SSSA Special Publication, 49)

PROJ ETO RADAMBRASIL. Folha SE. 22. Goiânia: geologia, geomorfologia, pedol ogia, vegetação e uso potencial da terra. Rio de J aneiro, 1983. 768p.

REID, J .B. \& GOSS, M.J . Changes in the aggregate stability of a sandy loam effected by growing roots of perennial ryegrass (Iolium-perenne). J. Sci. Food Agric., 31:325-328, 1980.

RICE, C.W.; MOORMAN, T.B. \& BEARE, M. Role of microbial biomass carbon and nitrogen in soil quality. In: DORAN, J.W. \& J ONES, A.J ., eds. Methods for assessing soil quality. Madison, Soil Science Society of America, 1996. p.203-215. (SSSA Special Publication, 49)

ROVIRA, A.D. Microbiology of pasture soils and some effects of microorganisms on pasture plants. In.: WILSON, J.R.,ed. Plant relation in pastures. Melbourne, CSIRO, 1978. p.95110.

SAS INSTITUTE. SAS User's guide: statistics. 5.ed. Cary, 1985. 956p.

SILVA-FILHO, G.N. \& VIDOR, C. As práticas de manejo de solo na população microbiana. R. Bras. Ci. Solo, 8:291-296, 1984.

SMITH, J.L. \& PAUL, E.A. The significance of soil microbial biomass estimations. In.: BOLLAG, J.M. \& STOTSKY, G. Soil biochemistry. New York, Marcel Dekker, 1990. p.357398.

SPARLING, G.P. Ratio of microbial biomass carbon to soil organic carbon as a sensitive indicator of changes in soil organic matter. Aust. J. Soil Res., 30:195-207, 1992.
TEIXEIRA, S.M.; MILHOMEM, A.; FREITAS, P.L. \& BLANCANEAUX, P. Microbacias hidrográficas e desenvolvimento rural. U ma abordagem socioeconômica. In: BLANCANEAUX, P., ed. Interações ambientais no Cerrado: microbacia piloto de Morrinhos, estado de Goiás, Brasil. Brasília, Empresa Brasileira de Pesquisa Agropecuária, 1998. p.261-282.

TURCO, R.F.; KENNEDY, A.C. \& J AWSON, M.D. Microbial indicators of soil quality. I n: DORAN, J.W.; COLEMAN, D.C.; BEZDICEK, D.F. \& STEWART, B.A., eds. Defining soil quality for a sustainable environment. Madison, Soil Science Society of America, 1994. p.73-90. (SSSA Special Publication, 35)

VANCE, E.D.; BROOKES, P.C. \& JENKINSON, D.S. An extraction method for measuring soil microbial biomass $C$. Soil Biol. Biochem, 19:703-707, 1987.

VARGAS, L.K. \& SCHOLLES, D. Biomassa microbiana e produção de $\mathrm{C}-\mathrm{CO}_{2}$ e N mineral de um Podzólico Vermel hoEscuro submetido a diferentes sistemas de manejo. R. Bras. Ci. Solo, 24:35-42, 2000.

VASCONCELLOS, C.A.; CAMPOLINA, D.C.A.; SANTOS, F.G.; EXEL PITTA, G.V. \& MARRIEL, I.E. Resposta da soja e da biomassa de carbono do sol o aos resíduos de cinco genótipos de sorgo. R. Bras. Ci. Solo, 23:69-77, 1999.

VENZKE FILHO, S.P. Microbiota e sua atividade em uma cronosseqüência sob sistema plantio direto. Piracicaba, Escola Superior de Agricultura Luiz de Queiroz, 1999. 65p. (Tese de Mestrado)

VETTORI, L. Métodos de análise de solo. Rio de J aneiro, Ministério da Agricultura, 1969. 24p. (Bol etim Técnico, 7)

WALKLEY, A. \& BLACK, I.A. An examination of the Degtjareff method for determinig soil organic matter and a proposed modification of the chromic acid titration method. Soil Sci., 37:29-38, 1934. 
A.F. D'ANDRÉA et al.

R. Bras. Ci. Solo, 26:913-923, 2002 